\title{
Postsynaptic localization of PSD-95 is regulated by all three pathways downstream ofTrkB signaling
}

\author{
Akira Yoshii ${ }^{1,2,3}$ * and Martha Constantine-Paton ${ }^{2,3,4}$ * \\ 'Department of Anatomy and Cell Biology, University of Illinois at Chicago, Chicago, IL, USA \\ ${ }^{2}$ McGovern Institute for Brain Research, Massachusetts Institute of Technology, Cambridge, MA, USA \\ ${ }^{3}$ Constantine-Paton Laboratory, Department of Brain and Cognitive Science, McGovern Institute for Brain Research, Massachusetts Institute of Technology, \\ Cambridge, MA, USA \\ ${ }^{4}$ Department of Biology, McGovern Institute for Brain Research, Massachusetts Institute of Technology, Cambridge, MA, USA
}

\section{Edited by:}

Nancy Ip, The Hong Kong University of Science and Technology, Hong Kong

\section{Reviewed by:}

Clive R. Bramham, University of Bergen, Norway

Lucas Pozzo-Miller, The University of Alabama at Birmingham, USA

\section{*Correspondence:}

Akira Yoshii, Department of Anatomy and Cell Biology, University of Illinois at Chicago, 808 South Wood Street, Room 578 MC 512, Chicago, IL 60612, USA

e-mail: ayoshii@uic.edu;

Martha Constantine-Paton, Constantine-Paton Laboratory, Department of Brain and Cognitive Science, McGovern Institute for Brain Research, Massachusetts Institute of Technology, 77 Massachusetts Avenue, Building 46-4165, Cambridge, MA 02139, USA

e-mail: Mcpaton@mit.edu
Brain-derived neurotrophic factor (BDNF) and its receptor TrkB regulate synaptic plasticity. TrkB triggers three downstream signaling pathways; Phosphatidylinositol 3-kinase (PI3K), Phospholipase $\mathrm{C} \gamma(\mathrm{PLC} \gamma)$ and Mitogen activated protein kinases/Extracellular signalregulated kinases (MAPK/ERK). We previously showed two distinct mechanisms whereby BDNF-TrkB pathway controls trafficking of PSD-95, which is the major scaffold at excitatory synapses and is critical for synapse maturation. BDNF activates the PI3K-Akt pathway and regulates synaptic delivery of PSD-95 via vesicular transport Yoshii and ConstantinePaton, 2007). BDNF-TrkB signaling also triggers PSD-95 palmitoylation and its transport to synapses through the phosphorylation of the palmitoylation enzyme ZDHHC8 by a protein kinase C (PKC; Yoshii etal., 2011). The second study used PKC inhibitors chelerythrine as well as a synthetic zeta inhibitory peptide (ZIP) which was originally designed to block the brain-specific PKC isoform protein kinase $M \zeta(P K M \zeta)$. However, recent studies raise concerns about specificity of ZIP. Here, we assessed the contribution of TrkB and its three downstream pathways to the synaptic distribution of endogenous PSD-95 in cultured neurons using chemical and genetic interventions. We confirmed that TrkB, PLC, and PI3K were critical for the postsynaptic distribution of PSD-95. Furthermore, suppression of MAPK/ERK also disrupted PSD-95 expression. Next, we examined the contribution of PKC. While both chelerythrine and ZIP suppressed the postsynaptic localization of PSD95, RNA interference for PKM $\zeta$ did not have a significant effect. This result suggests that the ZIP peptide, widely used as the "specific" PKM may block a PKC variant other than PKM $\zeta$ such as $P K C \lambda / l$. Our results indicate that TrkB regulates postsynaptic localization of PSD-95 through all three downstream pathways, but also recommend further work to identify other PKC variants that regulate palmitoylation and synaptic localization of PSD-95.

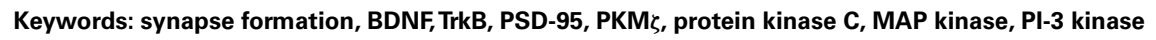

\section{INTRODUCTION}

The Brain-derived neurotrophic factor (BDNF) and its receptor TrkB are critical for maturation of both excitatory (Gorski et al., 2003; Wirth et al., 2003; Chakravarthy et al., 2006; Tanaka et al., 2008; Kaneko et al., 2012) and inhibitory neurons (Hanover et al., 1999; Huang et al., 1999). In the visual cortex, BDNF increases 2 weeks after birth largely due to the visual activity after eye opening (Castren etal., 1992). BDNF-TrkB signaling regulates excitation-inhibition balance (Schinder et al., 2000) and facilitates activity-dependent formation of neuronal circuits (Cabelli et al., 1995, 1997; Heimel et al., 2010) as well as critical period closure of ocular dominance (Hanover et al., 1999; Huang et al., 1999).

In excitatory neurons, BDNF-TrkB signaling regulates dendritic growth (Xu et al., 2000), spine maturation, stabilization (Gorski et al., 2003; Wirth et al., 2003; Chakravarthy et al., 2006; Tanaka et al., 2008; Kaneko et al., 2012), and long-term potentiation (LTP; Kang and Schuman, 1995; Figurov etal., 1996; Patterson etal., 1996; Tanaka et al., 1997; Frerking et al., 1998;
Gottschalk et al., 1998; Huber et al., 1998). BDNF-TrkB signaling also plays a critical role in the development of synapses by regulating the transport of the membrane associated guanylate kinase the post-synaptic density protein PSD-95. In the visual pathway of rodents upon eye-opening, PSD-95, which is the major scaffolding protein at mature glutamate synapses (Yoshii and Constantine-Paton, 2007; Yoshii et al., 2011), is transported to young synaptic contacts by BDNF/TrkB. SAP102, the dominant MAGUK in neonatal period, does not require an activation of BDNF-TrkB signaling for its postsynaptic localization (Yoshii and Constantine-Paton, 2007) and moves to early synapses in association with the NR2B subunit GluN2B (Washbourne et al., 2004). In our previous work, we reported two distinct mechanisms whereby the BDNF-TrkB pathway controls trafficking of PSD-95. BDNF activates the Phosphatidylinositol 3-kinase (PI3K)-Akt pathway and triggers synaptic delivery of PSD-95 via vesicular transport (Yoshii and Constantine-Paton, 2007). BDNF-TrkB signaling is also necessary for PSD-95's initial association with membranes. 
It initiates PSD-95 palmitoylation through the phosphorylation of the palmitoylation enzyme ZDHHC8 by Phospholipase $\mathrm{C} \gamma$ (PLC $\gamma$ ) and a protein kinase C (PKC; Yoshii et al., 2011). Here we verify our previous observations on the roles of the PI3KAkt and PLC $\gamma$ - PKC pathways in the transport of PSD-95 to synapses. We also extend these analyses of BDNF-TrkB signaling to the role of the third pathway downstream of TrkB activation: namely, the Mitogen activated protein kinase/Extracellular signalregulated kinase (MAPK/ERK) pathway. Finally, in light of recent publications indicating that ZIP, the reagent we used to block the $\mathrm{C}$ kinase PKM $\zeta$, is not specific for PKM $\zeta$ in LTP, (Lee et al., 2013; Volk et al., 2013) we test whether another PKC may also be involved in the palmitoylation of PSD-95.

\section{MATERIALS AND METHODS \\ ANIMALS}

TrkB ${ }^{\text {F616A }}$ mice carrying a modification in the ATP binding site of this kinase (Chen et al., 2005) were kindly provided by Dr. David Ginty. Wild type (WT) C57BL6 mice were obtained from Charles River Laboratories. All manipulations were performed in accord with the guidelines of the MIT-IACUC.

\section{PRIMARY NEURON CULTURE, LIPOFECTION, IMMUNOCYTOCHEMISTRY}

Occipital cortices of E15.5 mouse brains were dissected, digested with a solution containing papain and DNase for $25 \mathrm{~min}$. Cells were dissociated using fire polished glass pipets and plated at the density of $0.5 \times 10^{6}$ cells per $\mathrm{cm}^{2}$ after counting with a hemocytometer. Coverslips were coated with laminin and polyD-lysine. Transfections of DNA constructs encoding either small interfering RNA (siRNA) against PKM $\zeta$ or its scrambled sequence (kindly provided by Dr. Richard Huganir, Johns Hopkins University) was performed at day in vivo (DIV) 8 using Lipofectamine 2000 (Invitrogen) according to the manufacturer's protocol. On DIV15, cultured neurons were treated with the following reagents. 1NM-PP1 to block activation of TrkB or its control construct Bph-PP1 (kindly provided by Dr. Kevan Shokat, UCSF). U73122 (1 $\mu \mathrm{M})$, Chelerythrine $(2.5 \mu \mathrm{M})$, PD98059 (50 $\mu \mathrm{M})$, and Wortmannin (100 nM) were used to block PLC, PKC MAPK, and PI3K, respectively, and were purchased from Sigma-Aldrich. The PKM $\zeta$ inhibitory pseudosubstrate ZIP (myr-SIYRRGARRWRKL-OH) and scrambled peptide (myr-RLYRKRIWRSAGROH; Pastalkova et al., 2006) were synthesized in the MIT Biopolymers Laboratory.

Twenty-four hours after each exposure, cultures were fixed with $4 \%$ paraformaldehyde ( $15 \mathrm{~min}$ ). Neurons were permeabilzed with $0.3 \%$ TritonX-100 for $5 \mathrm{~min}$ and washed. Cultures were blocked with 9\% BSA ( $1 \mathrm{~h})$, and incubated with an antibody for PSD-95 (Neuromab \#K28/43, 1:1000) at room temperature overnight. After washing with PBS (3x), cultures were incubated with secondary antibody Alexa Fluor 488 (Molecular Probes) for $2 \mathrm{~h}$.

Images were captured using a $60 \mathrm{x}$ objective on a Nikon PCM 2000 confocal microscope and a $6-8 \mu \mathrm{m}, \mathrm{Z}$-series, of optical sections were taken at intervals of $0.5 \mu \mathrm{m} .12 / 4 \mu \mathrm{m}$ areas containing isolated typical secondary dendritic branches from pyramidal neurons were selected. For each area, a threshold was set to optimally represent PSD-95 puncta and exclude diffuse label in dendritic shafts (See Figure $2 \mathrm{~A}$ middle and bottom rows). The same threshold was applied to all neurons in the set of cultures in each experiment. Immunocytochemical experiments were repeated twice for each treatment condition. Eight cells from eight culture dishes were imaged. Using ImageJ, total pixel intensity was calculated as the sum of each pixel number multiplied by its intensity as measured above threshold for each cell. This value was then averaged across all dendritic segments sampled in the experiment and presented as total pixel intensity of PSD-95 puncta. Averaged cell body intensity was also measured in ImageJ by manually selecting soma (yellow circles in Figure 2A) and applying the Measure function in ImageJ. Subsequently, total pixel intensity of PSD-95 puncta was divided by the averaged cell body intensity for normalization.

\section{TUNEL ASSAY}

FragEL $^{\text {TM }}$ DNA Fragmentaion Detection Kit (Calbiochem) was used for the TUNEL assay. The procedures were performed according to the manufacturer's instructions. Cell numbers in $250 \mu \mathrm{m} \times 250 \mu \mathrm{m}$ were counted and the apoptosis incidence was calculated by dividing the TUNEL $(+)$ cell number with total cell number.

\section{STATISTICS}

A Student's $t$-test was used for comparison of two groups. Oneway ANOVA with post hoc Tukey tests were used for comparisons of more than two groups. $p<0.05$ were considered significant and indicated as $*$ in graphs. $p<0.01$ and $p<0.001$ are indicated as ${ }^{* *}$ and ${ }^{* * *}$. Numeric data are presented as average $\pm S D$ in the "Results" section. Error bars in graphs represent SEM.

\section{RESULTS}

We previously studied trafficking of PSD-95 in dendrites of cultured occipital cortical neurons using Fluorescent recovery after photobleaching (FRAP) and showed that bath application of BDNF as well as a BDNF-coated beads facilitated transport of GFP-tagged PSD-95 in dendrites (Yoshii and Constantine-Paton, 2007). Furthermore, BDNF application resulted in an increase of the intensity of PSD-95 immunolabeled puncta.

In the current experiments we first examined the postsynaptic distribution of PSD-95 in dissociated cultured neurons prepared from E15.5 cortices of TrkB ${ }^{\mathrm{F} 616 \mathrm{~A}}$ mice. This mouse strain has a single amino acid mutation in the intracellular domain of the TrkB receptor allowing the signaling activities of the receptor to be selectively blocked by the synthetic compound 1NM-PP (Chen et al., 2005). At DIV 15, we treated neurons with either 1NM-PP1 or the "control" non-blocking molecule Bph-PP1for $24 \mathrm{~h}$. Using the TUNEL assay, we examined the incidence of apoptotic cells at various concentrations of 1NM-PP1 (Figure 1). We found that the cell death incidence was not significantly different at $1(0.036 \pm 0.020$; total of 123 apoptotic cells out of 3430 cells in 33 images) and $3 \mu \mathrm{M}$ $(0.036 \pm 0.021$; total of 244 apoptotic cells out of 6652 cells in 33 images $)$ as compared with no treatment $(0.033 \pm 0.025$; total of 132 apoptotic cells out of 3961 cells in 33 images) (ANOVA relative the no treatment group or the control $\mathrm{Bph}-\mathrm{PP} 1$ group $p=0.96$ and 0.79 , respectively). We determined $9 \mu \mathrm{M}$ of $1 \mathrm{NM}-\mathrm{PP} 1$ is toxic because $40 \%$ of cells are TUNEL ${ }^{+}$. Consequently, PSD-95 puncta intensity was accessed using 1NM-PP1at $3 \mu \mathrm{M}$ or less. First, we 


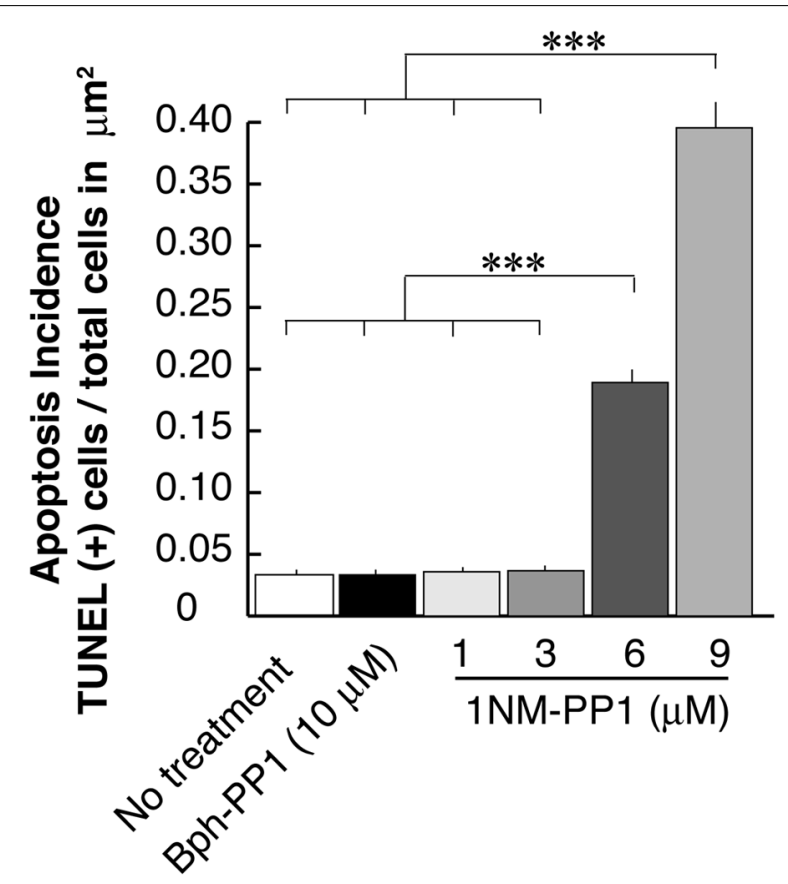

FIGURE 1 | Apoptosis rate of neurons that undergo TrkB signaling inhibition using the chemical genetic approach. Ratio's of Tunel signal positive cells to total cell number $(\sim 3,500-6,600$ cells examined in $250 \mu \mathrm{m} \times 250 \mu \mathrm{m}$ from eight cultures for each condition) are presented. Error bars represent SEM.

measured the averaged pixel intensity of the cell bodies (encircled areas with yellow dashed lines in Figure 2A) and found that PSD-95 expression level was slightly but significantly decreased in cultures treated with $2(41.5 \pm 7.6 ; N=8)$ or $3 \mu \mathrm{M}(41.5 \pm 8.7$; $N=8)$ of $1 \mathrm{NM}-\mathrm{PP} 1$ as compared with $1 \mu \mathrm{M}(50.4 \pm 10.1 ; N=8)$ or Bph-PP1 control ( $49.8 \pm 10.5 ; N=8)$. Therefore, we normalized the total pixel intensity of PSD-95 puncta by the intensity of soma. First we selected the defined area $(4 \mu \mathrm{m} \times 12 \mu \mathrm{m}$ squares in Figure 2A) of secondary dendritic branches and acquired total pixel intensities of PSD-95 puncta (See Materials and Methods; Yoshii and Constantine-Paton, 2007; Yoshii et al., 2011). These crude PSD-95 total pixel intensities were significantly reduced in neurons treated with 1NM-PP1 $(1 \mu \mathrm{M} ; 57,800 \pm 23,200 ; N=16)$ as compared to neurons without treatment $(23,100 \pm 11,600$; $N=16$; Figure 2C; ANOVA between $1 \mu \mathrm{M}$ and the no treatment groups; $p=0.0043$ ). Next, each intensity value was divided by averaged somal PSD-95 intensity of the same neuron. Normalized PSD-95 total pixel intensities remained significantly reduced in neurons treated with 1NM-PP1 as compared to control neurons treated with Bph-PP1 (Figure 2D; ANOVA between $1 \mu \mathrm{M}$ and the control groups; $p=0.0089)$. Higher concentration of 1NM-PP1 caused more suppression of PSD-95 puncta (ANOVA between 1 and $3 \mu \mathrm{M}$ groups; $p=0.043$; Figure 2D).

To examine each of the three TrkB downstream signaling pathways, we used antagonists against PLC, PI3K, and MAPK/ERK (Figure 3). First, we examined the PSD-95 immunolabel intensities of the somata and found that the blocker of the MAPK/ERK $(38.3 \pm 6.2 ; N=8)$ but not two other signaling pathways (PI3K;
$49.7 \pm 7.4 ; N=8$ and PLC; $45.5 \pm 4.7 ; N=8)$ resulted in a decrease of somal PSD-95 expression (Control; 49.9 $\pm 10.5 ; N=8$; Figure 3A). Next we examined PSD-95 total puncta intensities in dendrites (Figure 3B). As expected from our previous works, blockade of PLC with U73122 (31,700 $\pm 10,200 ; N=16)$ or PI3K with Wortmannin $(23,100 \pm 11,600 ; N=16)$ caused suppression of PSD-95 puncta intensities as compared to the no treatment controls $(50,900 \pm 35,100 ; N=16$; ANOVA; $p=0.042$ and 0.025, respectively). Furthermore inhibition of the MAPK/ERK pathway with PD98059 $(21,300 \pm 19,500 ; N=16)$ resulted in a decrease of PSD-95 puncta intensities (ANOVA to the no treatment group; $p=0.0017)$. Normalization to averaged cell body intensity also confirmed that all three downstream pathways were involved in the normal postsynaptic delivery and/or expression of PSD-95 (Figure 3C).

We previously showed that activation of PLC by BDNF-TrkB signaling is necessary for PSD-95 palmitoylation and its transport to synapses. This mechanism depends on the phosphorylation of the palmitoylation enzyme ZDHHC8 by a PKC (Yoshii et al., 2011), which is activated by PLC. In that study, we used the PKC inhibitor Chelerythrine as well as a synthetic PKM $\zeta$ inhibitory peptide ZIP which was originally thought to specifically block this brain-specific PKC isoform (Ling et al., 2002). We confirmed that ZIP treatments resulted in a reduction of PSD-95 puncta intensity $(25,100 \pm 13,900 ; N=16$; Figure 4A; ANOVA to control; $p=0.02$ ) as was also reported in the hippocampus (Shao et al., 2012), subsequent to our original finding in the visual cortex. Chelerythrine treatment also showed a similar result as ZIP treatment $(28,000 \pm 13,000 ; N=16$; ANOVA to control; $p=0.035$ ).

However, in contrast to earlier experiments suggesting that ZIP was a specific $\mathrm{PKM} \zeta$ antagonist (Ling et al., 2002), recent studies using PKM $\zeta$ knockout mice have not shown the expected LTP defect even though they did confirm that ZIP suppressed the LTP response (Lee et al., 2013; Volk et al., 2013). These reports raised the concern about the specificity of ZIP for PKM $\zeta$. Consequently, we tested PKM $\zeta$ directly by suppressing its expression using RNAi. We transfected DNA constructs encoding siRNA against PKM $\zeta$ or the scrambled sequence at DIV8 and examined it at DIV16. RNAi for PKM $\zeta$ did not have a significant effect on postsynaptic localization of PSD-95 (30,600 $\pm 21,200 ; N=16)$ as compared to neurons transfected with the scrambled sequence construct $(33,600 \pm 20,300 ; N=16$; Student's $t$-test; $p=0.73$; Figure 4B).

\section{DISCUSSION \\ SYNAPTIC EXPRESSION OF PSD-95 DEPENDS ON PKC BUT NOT PKM $\zeta$}

In our previous in vitro study, we applied BDNF to neurons expressing PSD-95 tagged with GFP and saw an increase in puncta intensity in $60 \mathrm{~min}$ (Yoshii and Constantine-Paton, 2007). We also used FRAP and pharmacological treatments and showed that the BDNF effect on vesicular transport of PSD-95 requires the PI3K/Akt pathway (Yoshii and Constantine-Paton, 2007). In the following in vivo study, we used $\operatorname{TrkB}^{\mathrm{F} 616 \mathrm{~A}}$ mice and showed that our synchronized eye-opening regime, which triggers PSD-95 redistribution to synapses in rats (Yoshii et al., 2003), also activates $\operatorname{TrkB}$ in visual cortex and regulates the synaptic 

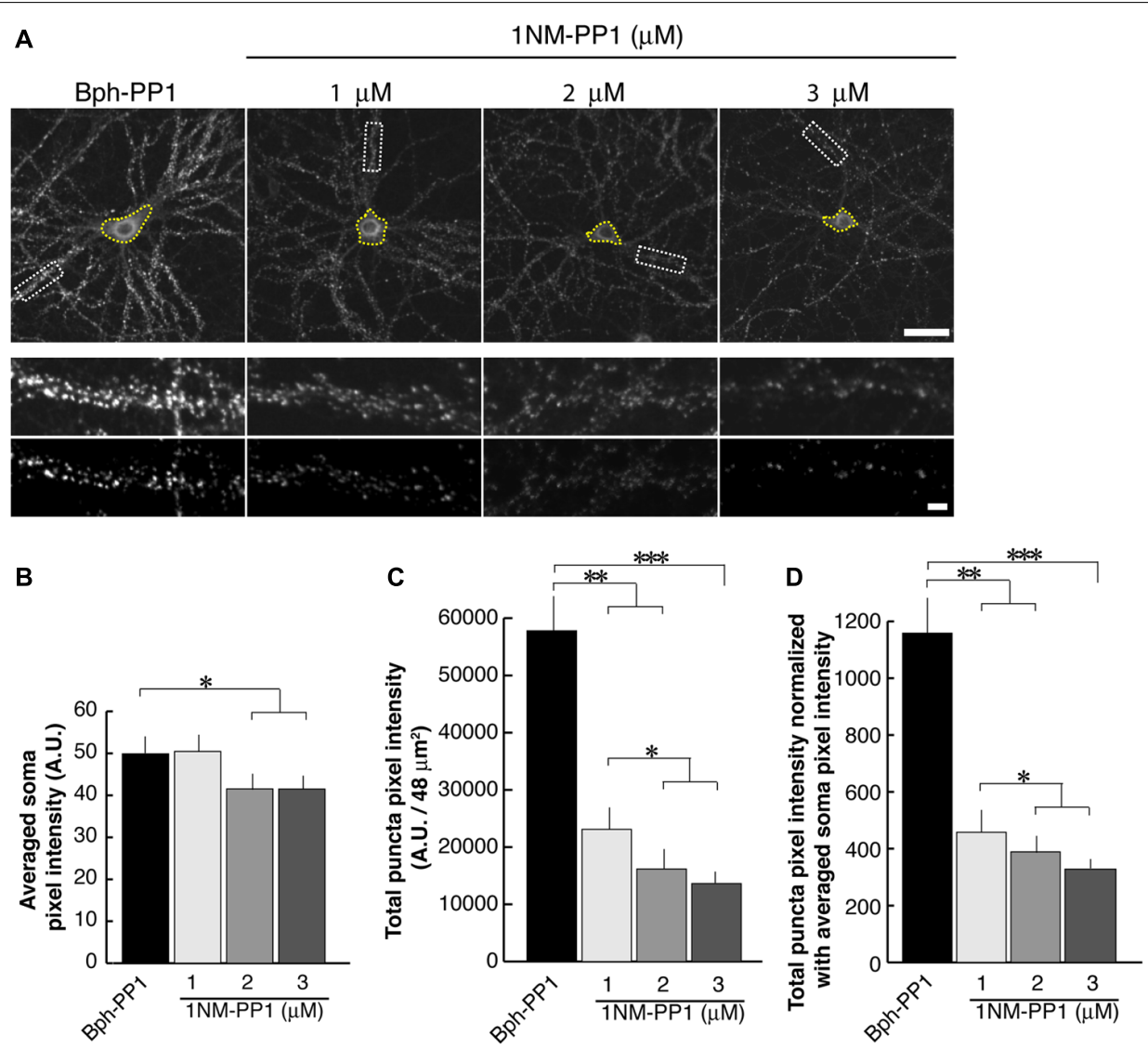

FIGURE 2 | 1NM-PP1 suppresses punctate distribution of PSD-95 dose-dependently in TrkB ${ }^{\mathbf{F 1 6 A}}$ neurons. (A) The top row shows representative neurons immunostainted with anti-PSD-95. Typical secondary dendritic branches analyzed are shown in $48 \mu \mathrm{m}^{2}$ rectangles in the middle row. These branches are processed after the thresholding shown in the bottom row. The scales in the top and bottom rows show 10 and $1 \mu \mathrm{m}$, respectively. (B) Graph showing averaged PSD-95 intensities in somata. (C) Graph showing quantification of PSD-95 puncta total pixel intensities. Note that all three concentrations of 1NM-PP1 result in reduced total PSD-95 puncta intensity. (D) In this graph, PSD-95 puncta intensities are normalized to somal PSD-95 intensities. In each condition, 16 branches from eight cells (chosen from the two different dissociations) were analyzed. Error bars represent SEM. localization of PSD-95. Furthermore, the TrkB activation is necessary for palmitoylation of PSD-95 in somata through PLC, PKC, and the palmitoylation enzyme ZDHHC8 (Yoshii etal., 2011). In the current study on cultured visual cortical neurons using the same pharmacological intervention, we confirmed that postsynaptic distribution of PSD-95 depends on BDNF-TrkB signaling, and that PI3K and PLC are necessary for increased PSD-95 at synaptic puncta. However, even though ZIP suppressed PSD-95 puncta intensity, knockdown of PKM $\zeta$ using RNAi has no effect on postsynaptic expression of PSD-95. This result is consistent with recent reports showing $\mathrm{PKM} \zeta$ knockout mice do not show an abnormal LTP response (Lee et al., 2013; Volk et al., 2013). One possibility for these LTP results, suggested by Lisman (2012), is that the concentration of ZIP is too high and causes non-specific inhibition (Lisman, 2012). Alternatively, ZIP may not be a specific PKM $\zeta$ inhibitor rather it may also interact with other PKC variants such as another atypical PKC (PKC $\lambda /$; Lee et al., 2013; Volk et al., 2013). These results necessitated revising our previous interpretation (Yoshii etal., 2011). The current results suggest that a PKC variant other than PKM $\zeta$ should be present in the developing visual cortex and is likely to phosphorylate the palmitoylation enzyme ZDHHC8.

\section{MAPK/ERK IS INVOLVED IN SYNAPTIC EXPRESSION OF PSD-95}

The current work also indicates that the MAPK/ERK pathway plays a role in the synaptic expression of PSD-95 as its inhibitor PD98059 significantly suppresses PSD-95 intensities of the soma as well as dendrite. This is consistent with the previous report showing that BDNF-induced increase in dendritic spine density is mediated by MAPK/ERK1/2 (Alonso et al., 2004). MAPK/ERK regulates protein-synthesis dependent plasticity by increasing phosphorylation of eukaryotic initiation factor $4 \mathrm{E}$ (eIF4E), the 4E-binding protein 1 (4E-BP1) and ribosomal protein S6 (Kelleher et al., 2004; Klann and Dever, 2004), therefore this pathway may directly initiate translation of PSD-95 gene either in the cell bodies of young neurons or in response to local activity in dendritic spines.

Brain-derived neurotrophic factor also activates the elongation of translation, which is mediated by PI3K and MAPK/ERK (Inamura et al., 2005). These two kinases turn off the eukaryotic 
A$$
\text { A }
$$

B

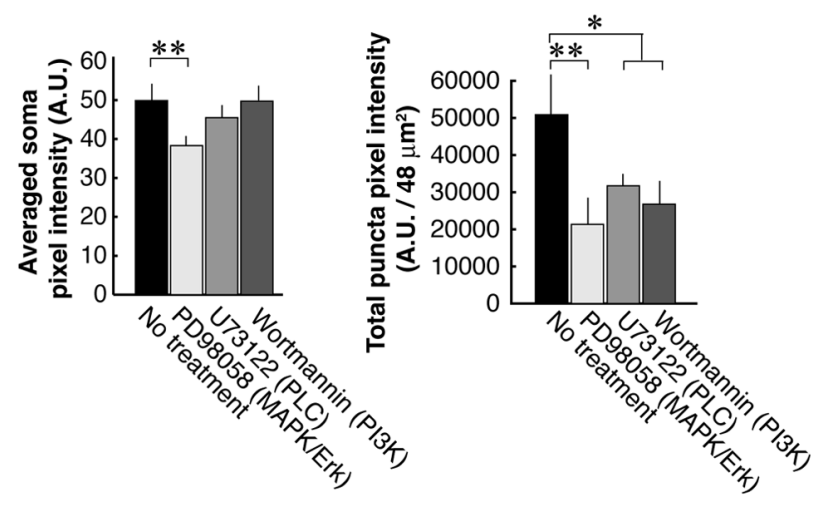

C

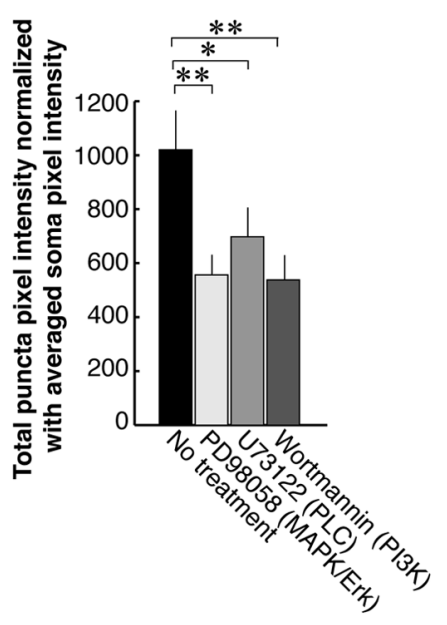

FIGURE 3 | PSD-95 puncta intensities in neurons that are treated with inhibitors of signaling molecules downstream of TrkB. Neurons are treated with blockers of the PLC (U73122, $1 \mu \mathrm{M})$, MAPK (PD98059, $50 \mu \mathrm{M})$, or PI3K (Wortmannin, $100 \mathrm{nM}$ ). Quantification was performed in the same manner as data presented in Figure 2. The graphs show averaged PSD-95 intensities in somata (A), total pixel intensities of PSD-95 puncta (B), and puncta intensities normalized to somal intensities (C). In each condition, 16 branches from eight cells were analyzed. Error bars represent SEM.

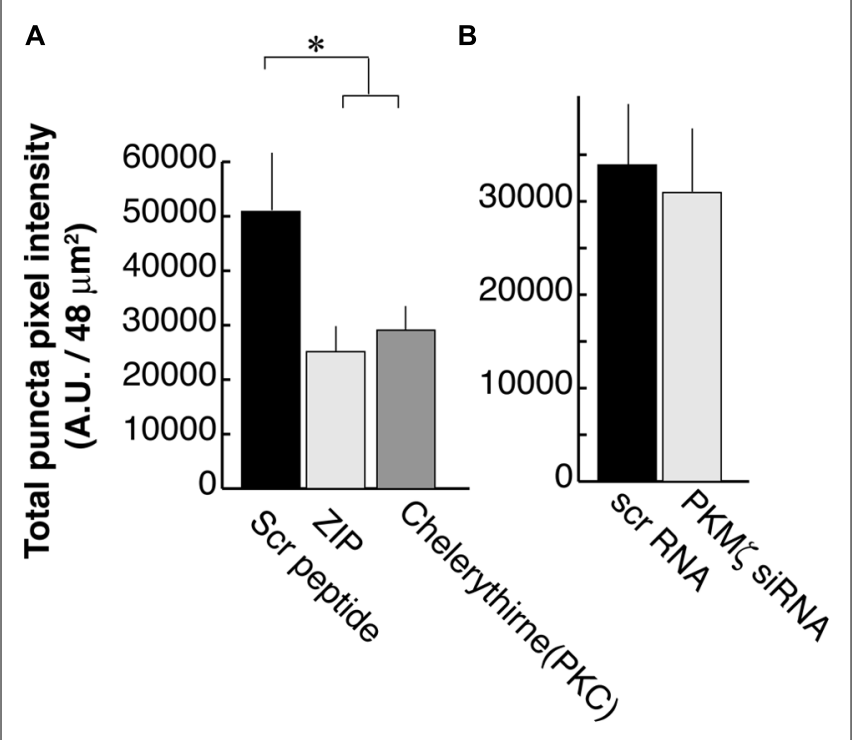

FIGURE 4 | Zeta inhibitory peptide (ZIP) but not PKM $\zeta$ knockdown causes suppression in PSD-95 puncta intensity. (A) Neurons are treated with ZIP $(1 \mu \mathrm{M})$, PKC (Chelerythrine, $2.5 \mu \mathrm{M})$, or the scrambled ZIP peptide $(1 \mu \mathrm{M})$. (B) Neurons are transfected with DNA constructs encoding siRNA for PKM $\zeta$ or the scrambled sequence. In both experiments, 16 branches from eight cells were analyzed. Error bars represent SEM.

elongation factor 2 kinase (eEF2K), in two parallel mechanisms. In turn, the suppression of eEF2K results in an increase of dephosphorylated and active eukaryotic elongation factor 2 (eEF2; Wang etal., 2001). This laboratory previously showed that NMDAR activation and visual activity rapidly induces phosphorylation of eEF2 at the synapse (Scheetz et al., 1997). This suppresses synthesis of most proteins but facilitates CaMKII synthesis. In rat dentate gyrus, BDNF application in vivo has been shown to induce LTP via ERK pathway, increase phosphorylation of eEF2 at non-synaptic sites and enhance expression of CaMKII- $\alpha$ and Arc (Kanhema et al., 2006). Furthermore, Worley and his colleagues obtained similar results for eEF2 phosphorylation via activation of mGluR5 in the hippocampus where the general shutdown of protein synthesis at the synapse significantly increased Arc/Arg3 translation (Park et al., 2008).

Another potential mechanism is that MAPK/ERK may be involved is mRNA transport. PSD-95 transcripts have been shown to exist in dendrites and interact with the fragile $\mathrm{X}$ mental retardation protein (FMRP). This enhances stability of the PSD-95 transcript and represses its translation during mRNA transport (Zalfa etal., 2007). A recent study shows that FMRP forms a complex with CYFIP1, a newly identified 4E-binding protein, and represses translation during mRNA transport (Napoli et al., 2008). BDNF can release this translational repression (Napoli et al., 2008). Whether MAPK/ERK meditates BDNF-dependent protein synthesis via translation and/or mRNA transport awaits future studies.

Brain-derived neurotrophic factor application also regulates transcription via MAPK/ERK which phosphorylates the CAMPresponse element binding transcription factor (CREB) at serine133 residue (Bonni etal., 1995, 1999; Finkbeiner et al., 1997; Shaywitz and Greenberg, 1999; Pizzorusso et al., 2000; Ying etal., 2002). Interestingly, CREB can activate the $B d n f$ gene through promoter IV (Hong etal., 2008) and amplify BDNF-dependent synapse maturation. Therefore, we predict that MAPK/ERK regulates either or both transcription and translation of PSD-95. However, it remains to be studied whether CREB directly activates transcription of PSD-95 itself or upregulates $\mathrm{BDNF}$, which further facilitates posttranscriptional regulation of PSD-95, i.e., translation, palmitoylation, or vesicular transport. 


\section{CONCLUSION}

It is now clear that the increases in PSD-95 at synapses are mediated by all three signaling pathways downstream of TrkB. They are involved in various processes regulating protein expression. MAPK/ERK could regulate transcription through CREB and other transcription factors. MAPK/ERK and PI3K-Akt pathway play major roles in translation (Kelleher et al., 2004; Klann and Dever, 2004). The PI3K pathway also facilitates vesicular transport of PSD-95 from ER to Golgi apparatus, then along microtubule. PSD-95 is synthesized in the cytoplasm and requires palmitoylation to become attached to membranes and to get delivered to postsynaptic membranes. This post-translational modification is regulated by BDNF-TrkB signaling via PLC-PKC. These same mechanisms are likely to regulate BDNF-dependent long-term plasticity. Furthermore, PSD-95 itself and its interaction with TrkB signaling have been implicated in various brain diseases, especially neurodevelopmental disorders such as autism spectrum disorders (Tsai et al., 2012), Angelman syndrome (Cao et al., 2013), and schizophrenia (Mukai et al., 2008).

\section{ACKNOWLEDGMENTS}

This work was supported by NIH grants 5R01EY006039-27 and 5R01EY014074-15. We thank Dr. Richard Huganir (Johns Hopkins University, Baltimore) for DNA constructs encoding small interfering RNA against $\mathrm{PKM} \zeta$ or the scrambled sequence, Dr. David D. Ginty (Johns Hopkins University, Baltimore) for the TrkB ${ }^{\text {F616A }}$ mouse strain, Dr. Kevan M. Shokat and Dr. Chao Zhang (UCSF, San Francisco) for 1NM-PP1 and Bph-PP1.

\section{REFERENCES}

Alonso, M., Medina, J. H., and Pozzo-Miller, L. (2004). ERK1/2 activation is necessary for BDNF to increase dendritic spine density in hippocampal CA1 pyramidal neurons. Learn. Mem. 11, 172-178. doi: 10.1101/lm.67804

Bonni, A., Brunet, A., West, A. E., Datta, S. R., Takasu, M. A., and Greenberg, M. E. (1999). Cell survival promoted by the Ras-MAPK signaling pathway by transcription-dependent and -independent mechanisms. Science 286, 1358-1362. doi: 10.1126/science.286.5443.1358

Bonni, A., Ginty, D. D., Dudek, H., and Greenberg, M. E. (1995). Serine 133phosphorylated CREB induces transcription via a cooperative mechanism that may confer specificity to neurotrophin signals. Mol. Cell. Neurosci. 6, 168-183. doi: 10.1006/mcne.1995.1015

Cabelli, R. J., Hohn, A., and Shatz, C. J. (1995). Inhibition of ocular dominance column formation by infusion of NT-4/5 or BDNF. Science 267, 1662-1666. doi: 10.1126/science.7886458

Cabelli, R. J., Shelton, D. L., Segal, R. A., and Shatz, C. J. (1997). Blockade of endogenous ligands of trkB inhibits formation of ocular dominance columns. Neuron 19, 63-76. doi: 10.1016/S0896-6273(00)80348-7

Cao, C., Rioult-Pedotti, M. S., Migani, P., Yu, C. J., Tiwari, R., Parang, K., et al. (2013). Impairment of TrkB-PSD-95 signaling in Angelman syndrome. PLoS Biol. 11:e1001478. doi: 10.1371/journal.pbio.1001478

Castren, E., Zafra, F., Thoenen, H., and Lindholm, D. (1992). Light regulates expression of brain-derived neurotrophic factor mRNA in rat visual cortex. Proc. Natl. Acad. Sci. U.S.A. 89, 9444-9448. doi: 10.1073/pnas.89.20.9444

Chakravarthy, S., Saiepour, M. H., Bence, M., Perry, S., Hartman, R., Couey, J. J., et al. (2006). Postsynaptic TrkB signaling has distinct roles in spine maintenance in adult visual cortex and hippocampus. Proc. Natl. Acad. Sci. U.S.A. 103, 1071-1076. doi: 10.1073/pnas.0506305103

Chen, X., Ye, H., Kuruvilla, R., Ramanan, N., Scangos, K. W., Zhang, C., et al. (2005). A chemical-genetic approach to studying neurotrophin signaling. Neuron 46, 13-21. doi: 10.1016/j.neuron.2005.03.009

Figurov, A., Pozzo-Miller, L. D., Olafsson, P., Wang, T., and Lu, B. (1996). Regulation of synaptic responses to high-frequency stimulation and LTP by neurotrophins in the hippocampus. Nature 381, 706-709. doi: 10.1038/381706a0
Finkbeiner, S., Tavazoie, S. F., Maloratsky, A., Jacobs, K. M., Harris, K. M., and Greenberg, M. E. (1997). CREB: a major mediator of neuronal neurotrophin responses. Neuron 19, 1031-1047. doi: 10.1016/S0896-6273(00)80395-5

Frerking, M., Malenka, R. C., and Nicoll, R. A. (1998). Brain-derived neurotrophic factor (BDNF) modulates inhibitory, but not excitatory, transmission in the CA1 region of the hippocampus. J. Neurophysiol. 80, 3383-3386.

Gorski, J. A., Zeiler, S. R., Tamowski, S., and Jones, K. R. (2003). Brain-derived neurotrophic factor is required for the maintenance of cortical dendrites. J. Neurosci. 23, 6856-6865.

Gottschalk, W., Pozzo-Miller, L. D., Figurov, A., and Lu, B. (1998). Presynaptic modulation of synaptic transmission and plasticity by brain-derived neurotrophic factor in the developing hippocampus. J. Neurosci. 18, 6830-6839.

Hanover, J. L., Huang, Z. J., Tonegawa, S., and Stryker, M. P. (1999). Brain-derived neurotrophic factor overexpression induces precocious critical period in mouse visual cortex. J. Neurosci. 19, RC40.

Heimel, J. A., Saiepour, M. H., Chakravarthy, S., Hermans, J. M., and Levelt, C. N. (2010). Contrast gain control and cortical TrkB signaling shape visual acuity. Nat. Neurosci. 13, 642-648. doi: 10.1038/nn.2534

Hong, E. J., Mccord, A. E., and Greenberg, M. E. (2008). A biological function for the neuronal activity-dependent component of Bdnf transcription in the development of cortical inhibition. Neuron 60, 610-624. doi: 10.1016/j.neuron.2008.09.024

Huang, Z. J., Kirkwood, A., Pizzorusso, T., Porciatti, V., Morales, B., Bear, M. F., et al. (1999). BDNF regulates the maturation of inhibition and the critical period of plasticity in mouse visual cortex. Cell 98, 739-755. doi: 10.1016/S00928674(00)81509-3

Huber, K. M., Sawtell, N. B., and Bear, M. F. (1998). Brain-derived neurotrophic factor alters the synaptic modification threshold in visual cortex. Neuropharmacology 37, 571-579. doi: 10.1016/S0028-3908(98)00050-1

Inamura, N., Nawa, H., and Takei, N. (2005). Enhancement of translation elongation in neurons by brain-derived neurotrophic factor: implications for mammalian target of rapamycin signaling. J. Neurochem. 95, 1438-1445. doi: 10.1111/j.14714159.2005.03466.x

Kaneko, M., Xie, Y., An, J. J., Stryker, M. P., and Xu, B. (2012). Dendritic BDNF synthesis is required for late-phase spine maturation and recovery of cortical responses following sensory deprivation. J. Neurosci. 32, 4790-4802. doi: 10.1523/JNEUROSCI.4462-11.2012

Kang, H., and Schuman, E. M. (1995). Long-lasting neurotrophin-induced enhancement of synaptic transmission in the adult hippocampus. Science 267, 1658-1662. doi: 10.1126/science.7886457

Kanhema, T., Dagestad, G., Panja, D., Tiron, A., Messaoudi, E., Havik, B., etal. (2006). Dual regulation of translation initiation and peptide chain elongation during BDNF-induced LTP in vivo: evidence for compartmentspecific translation control. J. Neurochem. 99, 1328-1337. doi: 10.1111/j.14714159.2006.04158.x

Kelleher, R. J. III, Govindarajan, A., Jung, H. Y., Kang, H., and Tonegawa, S. (2004). Translational control by MAPK signaling in long-term synaptic plasticity and memory. Cell 116, 467-479. doi: 10.1016/S0092-8674(04)00115-1

Klann, E., and Dever, T. E. (2004). Biochemical mechanisms for translational regulation in synaptic plasticity. Nat. Rev. Neurosci. 5, 931-942. doi: 10.1038/nrn1557

Lee, A. M., Kanter, B. R., Wang, D., Lim, J. P., Zou, M. E., Qiu, C., et al. (2013). Prkcz null mice show normal learning and memory. Nature 493, 416-419. doi: 10.1038 /nature 11803

Ling, D. S., Benardo, L. S., Serrano, P. A., Blace, N., Kelly, M. T., Crary, J. F., et al. (2002). Protein kinase Mzeta is necessary and sufficient for LTP maintenance. Nat. Neurosci. 5, 295-296. doi: 10.1038/nn829

Lisman, J. (2012). Memory erasure by very high concentrations of ZIP may not be due to PKM-zeta. Hippocampus 22, 648-649. doi: 10.1002/hipo.20980

Mukai, J., Dhilla, A., Drew, L. J., Stark, K. L., Cao, L., Macdermott, A. B., et al. (2008). Palmitoylation-dependent neurodevelopmental deficits in a mouse model of 22q11 microdeletion. Nat. Neurosci. 11, 1302-1310. doi: 10.1038/ nn. 2204

Napoli, I., Mercaldo, V., Boyl, P. P., Eleuteri, B., Zalfa, F., De Rubeis, S., et al. (2008). The fragile $\mathrm{X}$ syndrome protein represses activity-dependent translation through CYFIP1, a new 4E-BP. Cell 134, 1042-1054. doi: 10.1016/j.cell.2008.07.031

Park, S., Park, J. M., Kim, S., Kim, J. A., Shepherd, J. D., Smith-Hicks, C. L., et al. (2008). Elongation factor 2 and fragile $\mathrm{X}$ mental retardation protein control the 
dynamic translation of Arc/Arg3.1 essential for mGluR-LTD. Neuron 59, 70-83. doi: 10.1016/j.neuron.2008.05.023

Pastalkova, E., Serrano, P., Pinkhasova, D., Wallace, E., Fenton, A. A., and Sacktor, T. C. (2006). Storage of spatial information by the maintenance mechanism of LTP. Science 313, 1141-1144. doi: 10.1126/science.11 28657

Patterson, S. L., Abel, T., Deuel, T. A., Martin, K. C., Rose, J. C., and Kandel, E. R. (1996). Recombinant BDNF rescues deficits in basal synaptic transmission and hippocampal LTP in BDNF knockout mice. Neuron 16, 1137-1145. doi: 10.1016/S0896-6273(00)80140-3

Pizzorusso, T., Ratto, G. M., Putignano, E., and Maffei, L. (2000). Brain-derived neurotrophic factor causes cAMP response element-binding protein phosphorylation in absence of calcium increases in slices and cultured neurons from rat visual cortex. J. Neurosci. 20, 2809-2816.

Scheetz, A. J., Nairn, A. C., and Constantine-Paton, M. (1997). N-methyl$\mathrm{D}$-aspartate receptor activation and visual activity induce elongation factor-2 phosphorylation in amphibian tecta: a role for N-methyl-D-aspartate receptors in controlling protein synthesis. Proc. Natl. Acad. Sci. U.S.A. 94, 14770-14775. doi: $10.1073 /$ pnas.94.26.14770

Schinder, A. F., Berninger, B., and Poo, M. (2000). Postsynaptic target specificity of neurotrophin-induced presynaptic potentiation. Neuron 25, 151-163. doi: 10.1016/S0896-6273(00)80879-X

Shao, C. Y., Sondhi, R., Van De Nes, P. S., and Sacktor, T. C. (2012). PKMzeta is necessary and sufficient for synaptic clustering of PSD-95. Hippocampus 22, 1501-1507. doi: 10.1002/hipo.20996

Shaywitz, A. J., and Greenberg, M. E. (1999). CREB: a stimulus-induced transcription factor activated by a diverse array of extracellular signals. Annu. Rev. Biochem. 68, 821-861. doi: 10.1146/annurev.biochem.68.1.821

Tanaka, J., Horiike, Y., Matsuzaki, M., Miyazaki, T., Ellis-Davies, G. C., and Kasai, H. (2008). Protein synthesis and neurotrophin-dependent structural plasticity of single dendritic spines. Science 319, 1683-1687. doi: 10.1126/science.11 52864

Tanaka, T., Saito, H., and Matsuki, N. (1997). Inhibition of GABAA synaptic responses by brain-derived neurotrophic factor (BDNF) in rat hippocampus. J. Neurosci. 17, 2959-2966.

Tsai, N. P., Wilkerson, J. R., Guo, W., Maksimova, M. A., Demartino, G. N., Cowan, C. W., et al. (2012). Multiple autism-linked genes mediate synapse elimination via proteasomal degradation of a synaptic scaffold PSD-95. Cell 151, 1581-1594. doi: 10.1016/j.cell.2012.11.040

Volk, L. J., Bachman, J. L., Johnson, R., Yu, Y., and Huganir, R. L. (2013). PKM-zeta is not required for hippocampal synaptic plasticity, learning and memory. Nature 493, 420-423. doi: 10.1038/nature11802

Wang, X., Li, W., Williams, M., Terada, N., Alessi, D. R., and Proud, C. G. (2001). Regulation of elongation factor 2 kinase by p90(RSK1) and p70 S6 kinase. EMBO J. 20, 4370-4379. doi: 10.1093/emboj/20.16.4370
Washbourne, P., Liu, X. B., Jones, E. G., and Mcallister, A. K. (2004). Cycling of NMDA receptors during trafficking in neurons before synapse formation. $J$. Neurosci. 24, 8253-8264. doi: 10.1523/JNEUROSCI.2555-04.2004

Wirth, M. J., Brun, A., Grabert, J., Patz, S., and Wahle, P. (2003). Accelerated dendritic development of rat cortical pyramidal cells and interneurons after biolistic transfection with BDNF and NT4/5. Development 130, 5827-5838. doi: 10.1242/dev.00826

Xu, B., Zang, K., Ruff, N. L., Zhang, Y. A., Mcconnell, S. K., Stryker, M. P., et al. (2000). Cortical degeneration in the absence of neurotrophin signaling: dendritic retraction and neuronal loss after removal of the receptor TrkB. Neuron 26, 233245. doi: 10.1016/S0896-6273(00)81153-8

Ying, S. W., Futter, M., Rosenblum, K., Webber, M. J., Hunt, S. P., Bliss, T. V., et al. (2002). Brain-derived neurotrophic factor induces long-term potentiation in intact adult hippocampus: requirement for ERK activation coupled to CREB and upregulation of Arc synthesis. J. Neurosci. 22, 1532-1540.

Yoshii, A., and Constantine-Paton, M. (2007). BDNF induces transport of PSD-95 to dendrites through PI3K-AKT signaling after NMDA receptor activation. Nat. Neurosci. 10, 702-711. doi: 10.1038/nn1903

Yoshii, A., Murata, Y., Kim, J., Zhang, C., Shokat, K. M., and ConstantinePaton, M. (2011). TrkB and protein kinase M zzeta\} regulate synaptic localization of PSD-95 in developing cortex. J. Neurosci. 31, 11894-11904. doi: 10.1523/JNEUROSCI.2190-11.2011

Yoshii, A., Sheng, M. H., and Constantine-Paton, M. (2003). Eye opening induces a rapid dendritic localization of PSD-95 in central visual neurons. Proc. Natl. Acad. Sci. U. S. A. 100, 1334-1339. doi: 10.1073/pnas.0335785100

Zalfa, F., Eleuteri, B., Dickson, K. S., Mercaldo, V., De Rubeis, S., Di Penta, A., et al. (2007). A new function for the fragile $\mathrm{X}$ mental retardation protein in regulation of PSD-95 mRNA stability. Nat. Neurosci. 10, 578-587. doi: 10.1038/nn1893

Conflict of Interest Statement: The authors declare that the research was conducted in the absence of any commercial or financial relationships that could be construed as a potential conflict of interest.

Received: 10 January 2014; paper pending published: 09 February 2014; accepted: 10 March 2014; published online: 31 March 2014.

Citation: Yoshii A and Constantine-Paton M (2014) Postsynaptic localization of PSD95 is regulated by all three pathways downstream of TrkB signaling. Front. Synaptic Neurosci. 6:6. doi: 10.3389/fnsyn.2014.00006

This article was submitted to the journal Frontiers in Synaptic Neuroscience.

Copyright (C) 2014 Yoshii and Constantine-Paton. This is an open-access article distributed under the terms of the Creative Commons Attribution License (CC BY). The use, distribution or reproduction in other forums is permitted, provided the original author(s) or licensor are credited and that the original publication in this journal is cited, in accordance with accepted academic practice. No use, distribution or reproduction is permitted which does not comply with these terms. 